$$
\begin{aligned}
& \text { SLIC/NP- } 32 \\
& \text { DESJ } 001470
\end{aligned}
$$

\title{
INVESTIGATION OF A MONOCHROMATOR SCHEME FOR SPEAR*
}

\author{
K. WILE AND A. W. CHAO \\ Slanford Linear Accelerator Center \\ Stanford Universily, Stanford, California, 94905
}

\section{Introduction}

Because of the energy spread of the particles in an electron beam due to quantum fucluations the hadronic event rate at varrow resonas es is significantiy reduced. This effect may to some extent be reduced by use of a "monochromator scheme" as suggested by Renieri.' This schems requires a vertical disperaion in the interaction point but with differen! signs for the two beanis. If for jastance positrons have a positive dispersion the electron bave to bave a negative one. Under these condition positrons with the energy $E_{0}+\Delta E$ hit electrons witb $E_{0}-\Delta E$ and vice versa as sbown in Fig. 1 . Such a scheme makes if more likely that particle with the correct total energy $2 E_{0}$ collide in the interaction point.

The required particle dependent dispersion at the interaction poiut may be generated by use of a skew clectrostatic quadrupole mounted in a position with a horizontal dispersion. The skew quad couples the borizontal dispersion into the vertical plane with different sigos depending on the particle charge. This scheme requires two skew quadrupoles, one on each side of the interaction point. With a properly matched phase advance betwen these quads one can avoid a dispersion coupling in the rest of the storage ring.

*Work eupported by the Department of Energy, contract DE-AC03-765F00s15. 


\section{PORTIONS OF THIS REPORT ABE ULLEGBLE}

\section{it has been reproduced from the best availatile copy to permit the broadest pussible availabillty.}

Unfortunately, as demonstrated by H. Wiedemann, ${ }^{2}$ thin simple monochromator scheme does not improve the hadronic event rate. The reason is that with the skew quadrupoles not only the dispersion but also the betatron oscillations are coupled into the vertical plane, increasing the betatron beam oize $\sigma_{y}^{*}$ at the interaction point sigoificantly. Since the enhansement factor of the monochromator scheme is mainly determined by the ratio $\frac{\Delta_{p}}{p} \cdot \eta_{y}^{*} / \sigma_{y}^{*}$, where $\eta_{y}^{*}$ is the dispersion in the interaction point and $\Delta p / p$ is the beam energy spread, the hadronic event rate will approximately stay constant.

The way out is the more sophisticated monochromator scheme suggested at $\mathrm{N}$ suosibirsk, ${ }^{3}$ which uses four electrostatic quadrupoles at proper locations (-ig. 2). The first guad ESQ1 has in the vertical plane $90^{\circ}$ betatron phase advance to the interaction point and is mounted at a position with zero borizontal dispersion. The second quad (ESQ2) has a position with a aufficiently large borizoutal dispersion. The betatron phase between the two electrostatic skew quads shouid be 180 degrees in both plares.

The skew quadrupole ESQ2 is used to couple the horizontal dispersion into the vertical plane generatiog the required vertical dispersion $\eta_{y}^{*}$ in the interaction point. Because of optical reasons the vertical dispergion passes through ESQ1 on the axis and is therefore not affected by ESQ1. The coupled betatron oscillation, however, can be compensated by setting $E S Q 1$ to a proper value which je given by

$$
E S Q 1=-E S Q 2 \sqrt{\frac{\beta_{x 1} \cdot \beta_{y 1}}{\beta_{x 2} \cdot \beta_{y 2}}}
$$

where $\beta$ 's refer to the $\beta$-functions at ESQ1 and ESQ2. When the betatron coupling is compensated, the resulting betatron benm size $\sigma_{y}^{*}$ becomes very small, thus overcoming the dificulty mr.mintered in the scheme using only one pair of skew quads.

wenerally with surh a monochromator scheme one gets an increase of the 
hadronic event rate for a given luminosity of

$$
\lambda=\sqrt{1+\left(\frac{\eta_{y}^{*}}{\sigma_{y}^{*}} \cdot \frac{\Delta p}{p}\right)^{2}}
$$

The aim of the study presented in this paper is to investigate the possibjilty of inserting a monochromator scheme vith compensation of the betatron coupling in SPEAR.

\section{Monochromator Scheme With The Mini- $\beta$ ' SPEAR Lattice}

We have first looked at the possibility to insert the monochromator acheme into the recently implemented SPEAR mini beta lattice." Ar tually there are locations for the electrostatic skew quads satisfying approximately the monochromator constrainte mentioned above (Fig. 3). ESQ1 is mounted close to the horizontal focusing quadnupole $Q 2$ and $F S Q 2$ between $Q F 2$ and $Q F$. Both places bave enough space for an electrostatic quadrupole of $2 m$ length." There is no borizontal dispersion in the $E S Q 1$ region whereas between $Q F 2$ and $Q F$ the dispersiop has a relatively large value of $\eta_{x}=2.61 \mathrm{~m}$. The betatron phase advance between the two electrostatic skew quads is $\Delta \psi_{s}=202$ degree and $\Delta \psi_{y}=158$ degree in the horizontal and vertical planes respectively. This doesn't fulfill exactly the 180 degree constrajnt but may be sufficient to get a small vertical beam size $\sigma_{y}^{*}$ at the interaction point. The phase between ESQ1 and the interaction point is $\Delta \psi_{y}=96$ degree which is close to the correct value. In fact an altempt was made to produce a lattice strictly satisfjing these phase conditions by varying the quadrupole strengths. This has not produced any surprises and the results described below essentially stay unchanged.

- We will show later that the $2 \mathrm{~m}$ space is not absolutely eritical. The elcetrostatic skew quads can have a shorter length, perhaps $1 m$ would suffice. 
In order to calculate the dispersion $\eta_{\text {; }}$ at the interaction point one has to estimate the maximutn available gtrength of an electrostatic quadrupole which io given by the following relation

$$
k=2 \times 10^{-9} \frac{U}{E \cdot R^{2}}
$$

If $U$ is the roltage between one pole and ground in volts, $E$ the beam energy in $G e V$ and $R$ the pole radius in $m$, the quadrupole strength $k$ is given in $m^{-2}$. Since electrostatic devices are generally very weak compared to normal tron magnets, the pole radius $R$ should be chosen to be tmall. On the other hand the skew quads must not reduce the beam aperture which is supposed to be at least $\pm 12 \cdot \sigma$. Under these condition the minimum pole radius of $E S Q 2$ is $R=0.049 \mathrm{~m}$. The upper limit of the voltage is of the order of $V_{\text {max }}=100 \mathrm{hV}$. With these values the resulting quadrupole strength at the $\psi^{\prime}$-resonance $(E=1.89 \mathrm{GeV})$ becomes $k=0.044 m^{-2}$.

The skew quad $E S Q^{2}$ geverates a vertical diapersion which starts at this polat with an angle

$$
n_{y}^{\prime}=k \ell_{x}
$$

where $\eta_{\varepsilon}$ is the horizontal dispersion at $E S Q 2$ and $k-\ell$ is the integrated quadrupole streagth. The vertical dispersion in the interaction point is provided by the following transformation

$$
\eta_{y}^{*}=\eta_{y}^{*} \sqrt{\beta_{y}^{*} \beta_{y}} \sin \phi_{y}
$$

In this equation $\beta_{y}^{*}$ and $\beta_{y}$ are the vertical beta function at the interaction point 
and at the location of ESQ2 respectively. With the values

$$
\begin{aligned}
k & =0.044 \mathrm{~m}^{-2} \\
\ell & =2.0 \mathrm{~m} \\
\eta_{x} & =2.61 \mathrm{~m} \\
\beta_{y} & =4.79 \mathrm{~m} \\
\beta_{y}^{*} & =0.03 \mathrm{~m} \\
\psi_{y} & =4.43 \mathrm{rd}
\end{aligned}
$$

one can calculate the vertical dispersion to be

$$
\eta_{j}^{*}=-0.084 m
$$

which seems resonably large. The atrength o: ESQ1 given by E4. (1) is $k=$ $-0.013 m^{-2}$ asuming again $\ell=2 m$. The betatron beam size which is given by the relation

$$
\sigma_{y}^{\bullet}=\sqrt{\epsilon_{y} \beta_{y}^{*}}
$$

requires the knowledge of the vertical bean emittance $\epsilon_{\boldsymbol{y}}$. This erpittance results Iron: quantum excitatios due to aynchrotron radiation in the bendiog magnets where $\eta_{y} \neq 0$. In a machine like SPEAR witb a constanl bending radius $\rho$ for all magnets the emittance may be expressed as

$$
\epsilon_{y}=\frac{c \cdot E^{2}}{\rho \cdot \ell_{b}} \int_{\text {brends }} H_{y}(s) d s
$$

with

$$
H_{y}(s)=\gamma_{y} \eta_{y}^{2}+2 \alpha_{y} \eta_{y} \eta_{y}^{\prime}+\beta_{y} \eta_{y}^{n}
$$

The congtant $a$ has the value $a=1.4675 \times 10^{-6} \mathrm{~m} / \mathrm{GeV}^{2}$ and $\ell_{b}$ is the total orbit length inside all bending magnets around the ring. Since the vertical dispersion is nonzero only in the interaction region between the two skew quads ESQ2 the integral in (7) has only to be calculated over all bendin ${ }_{6}$ magnets in this region. 
Since there is no vertical bending in SPEAR, the coupled vertical dispersion bebaves like a normal particle trajectory. In particulat the function $\boldsymbol{H}_{*}$ (s) nepresenting the surface of the phase ellipse is a constant in the interaction region. Therefore one can express $H_{y}(s)$ by taking the optical values in the interaction point wbich reduces $(B)$ to

$$
H_{v}(s)=\frac{\eta_{p}^{*}}{\beta_{i}^{*}}=\text { const. }
$$

With this expression the vertical enittance becomes

$$
c_{y}=\frac{a E^{2} \eta_{y}^{2}}{\rho \beta_{y}^{*}} \cdot \frac{\epsilon_{1}}{b_{b}}
$$

where $\ell_{7}$ is the sum of the orbit length in all begdiog magnets with nonzero vertical dispersion.

In the SPEAR mini beta lattice there are thrce normal and one balf bending magoets on either side of the interaction point between the twc ESQ2's. Providing that the monochromator scbeme is installed onjy in one interaction region we get $\ell_{7}=7 \cdot \ell_{0}$ where $\ell_{0}=2.36825 \mathrm{~m}$ is the total length of one normat bending magnet. The total length of all SPEAR bends is $\boldsymbol{q}_{6}=34 \cdot \boldsymbol{c}_{0}$. The resulting vertical emittance at $E=1.89 \mathrm{GeV}$ is

$$
c_{y}=\frac{\beta}{\rho \beta_{y}^{2}} \frac{7}{34}=1.98 \times 10^{-8} \pi \mathrm{m}-\mathrm{rad}
$$

with the beading radius $\rho=12.815 \mathrm{~m}$. The horizontal emittance of the SPEAR mioi beta opties at the azme energy is $\epsilon_{x}=1.786 \times 10^{-7} \pi \mathrm{m} \cdot \mathrm{rad}$ which gives the emittaoce ratio of $\epsilon_{y} / \epsilon_{y}=0.11$.

Taking th: expressions (2), (6) and (10) the ephancement factor due to the 
monuchromatization finally becomes

$$
\lambda=\sqrt{1+\frac{\rho \cdot C_{b}}{a+B^{2}}\left(\frac{\Delta P}{p}\right)^{2}}
$$

which gives the value for the unchanged SPFAK stini beta tattice of

$$
\lambda=1.85
$$

with the evargy spread $A p / p=4.52 \times 10^{-4}$ at $E \cdot 1.89 \mathrm{GeV}$.

In this calculation the vertical emittance is only determined by quantum fuctuations, all other effects at resilual betatron coupling etc. have been neglected. This would slightly reduce the $\lambda$ - value. It is interesting to meatic a that under thit condition the enhancement factor doesn't depend on the optical dala $\eta_{j}^{;}$and $\beta_{y}^{*}$ 2t the interaction point. Thereforc they are not thit suitable parameters to vary in order to optimize the rel-tively low cachascement factor of $\lambda=1.85$. Note that $\lambda$ in this care is also independed of the besm exergy since $\Delta p / p \sim E$. Note also that since $\lambda$ is inderendent of $\eta_{j}^{*}$, the ekew quads only bave to be strong enough that the resulting $\frac{\Delta_{y}}{p} \cdot \eta_{j}^{*}$ is much larger than the vertical bear aixe corn* ing from residual betatim coupling. This means the olew quad strength can be reduced or their length abortened somewhat to accomodate practical considerations. Ope such consideration is that the space suggented for ESQ2 preseptly also contains the injection kicher. By shorteping ESQ2, it might be possible to have hoth ESQ2 aud the kicker in the available free apace. 


\section{Investigation of a Particular Monochromator Lattice for SPEAR}

Since for the given miri beta optics the increase of the hadronic event rate cn a narrow resonance due to monochromatization is only a factor of two, we farther investigated the possibility of removing the restrictions of keeping the magnet configuration unchanged. We have thus studied those configurations in whith the first vertically focusing quadrupole $Q 3$ bas been left in its position so that no modification of the particle detector MARK UI is necessary.

A particular monochromator optics has to ant ${ }^{*}$ y the following constraints:

1. The aumber and strength of bending magnets in the region with vertical dispersion should be as small as possible. On the other hand at least one magnet is required to get a zero horizontal diapersion at the first glew quad ESQ1.

2. The development of beam waists in both planes between ESQ1 and ESQ2 provides the required $\mathbf{1 8 0}$ degree phase advance.

3. In order to get a sufficjently large vertical dispergion $\eta_{y}^{*}$ in the interaction point the ho:izontal dispersion at the kkew quad ESQ2 should be atso suficiently large.

Based on these three constraints a monochromator optics for SPEAR bas been designed which is shown in Fig. 4. Only one weak beuding magnet with the standard length $\ell_{0}$ but only balf of the field strength of the normal bends is installed between ESQ1 and ESQ2. This provide: because of a relatively long drift space a horizontal dispersion of $\eta_{x}=1.2 \mathrm{~m}$ it $E S Q 2$ The geometry of the storage ring is changed from the existing one but the circumference bas been kept hxed.

Since the weak bending magnet has a radius $\rho_{w}=2 \cdot \rho$ th emittance formula has to be modified. In this case is

$$
\epsilon_{y}=a \cdot E^{2} \cdot H_{y}(s) \frac{\epsilon_{y} / \rho_{s}^{3}}{\left(2 \ell_{4} / p_{4}^{2}\right)+\left(4 / \rho^{2}\right)}
$$


where $\boldsymbol{\ell}_{n}=2 \boldsymbol{\ell}_{0}$ is the length of the two weak bending magnels and $\boldsymbol{\ell}_{6}=30 \boldsymbol{\ell}_{0}$ the total length of all other magnets around the ring. Actually there are four weak bending magnets installed in the ring namely one per quadrant but only two of them contribute to the vertical emittance. After some manjpulations considering the relation (9) the emittance becones

$$
A_{y}=\frac{a \cdot E^{2} \cdot \eta_{y}^{\prime \prime}}{124 \cdot \rho \cdot \beta_{y}^{\prime}}
$$

The resulting enhancement factor for this particular monocbromator optics is

$$
\lambda=\sqrt{1+\frac{124 \rho}{a \cdot E^{2}} \cdot\left(\frac{\Delta p}{p}\right)^{2}}
$$

The bending radius for this lattice is $\rho=12.061 \mathrm{~m}$ and the energy spread is $\Delta p / p=4.65 \times 10^{-4}$ at $E=1.89 \mathrm{GeV}$. With these values we finally obtain an enhancement factor

$$
\lambda=\mathbf{7 . 8 5}
$$

which is a significant improvement of the hadronic event rate.

Unfortunately we found that it was not possible to match this monochromator section properly to the rest of the ring. Even significant modifications in the arcs and the addition of more independent quadrupole families couldn't provide a satisfactory solution. In particular the chromaticity rose to values of more than $\xi=-30$ in both planes which is too large to be compensated in a small machine like SPEAR without reduction of the dynamic aperture. Both the high chromaticity and the extremely critical optics as fougd in computer caleulations makes it unlikely that this optics will properly work in a real machine. Reduced maximum beam currents and a much more unreliable operation nuy easily cancel the enhancement factor. Therefore for this small machine SPEAR this pasticular mononchromator insertion doesn't 'sem to be a resonable solution. For a larger 
machine, bowever, with longer straight gections which allow better matching to the arcs, the scheme described in this section may improve the hadronic event rate at a resonance considerably.

\section{Summary}

We investigated the possibility of mono-chr mmatizing SPEAR for the purpose of increasing the badronic event rate at the narr of eloctrestatic skew quads in the monochromator scheme it is found that the event rate can be increase by a factor of 2 for the mini beta optics assuming the luminosity is kept unchanged. An attempt to increase this enhancement factor by major rearrangements of the ring maguets has encountered serious optical difficulties; although enhancement factor of 8 sems possible in principle, this alternative is not recommended.

\section{Acknowledgement.}

We would like to thank our colleagues M. Allen, P. Morton, A. Hofmann, H. Wiedemann, R. Ruth and J. Paterson for several very useful discussions. D. Coward bas encouraged us and joined discussions in persuing these studies. One of us (K. W.) would tike to express his gratitude toward his SLAC colleagues for the enjoyable year he spent there.

\section{References}

1. A. Renieri, Frascati, Preprint / INF-75 / 6 (R), 1975.

2. H. Wiedemand, "Is it Possible to Improve the Event Rate for Narnow Resonances in SPEAR and PEP?," SLAC/PEP Note-320, October 1979.

3. A. A. Avdienko et al., "The Project of Modernizntion of the VEPP-4 Storage Ring for Monochromatic Experiments in the Energy Range of $\psi$ and $\Upsilon$ Mesons," Proceedinga 12 ${ }^{\text {h }}$ Intera. Conf. on High Energy Accelerators, Fermilab, 1983, p. 186.

4. K. Wi..e, “A Proposed Miai Beta Insertion for SPEAR," SLAC/AP-9, March 1984. 


\section{Figuse Captions}

I. Basic coucept of the monochromator.

2. Monochromator scheme with compensation of the betatron coupling.

3. Insertion of the monochromator acbeme into the unchanged SPEAR mini beta lattice.

4. Design of a particular monochromator optics fer SPEAR. 
Monochromator scheme:
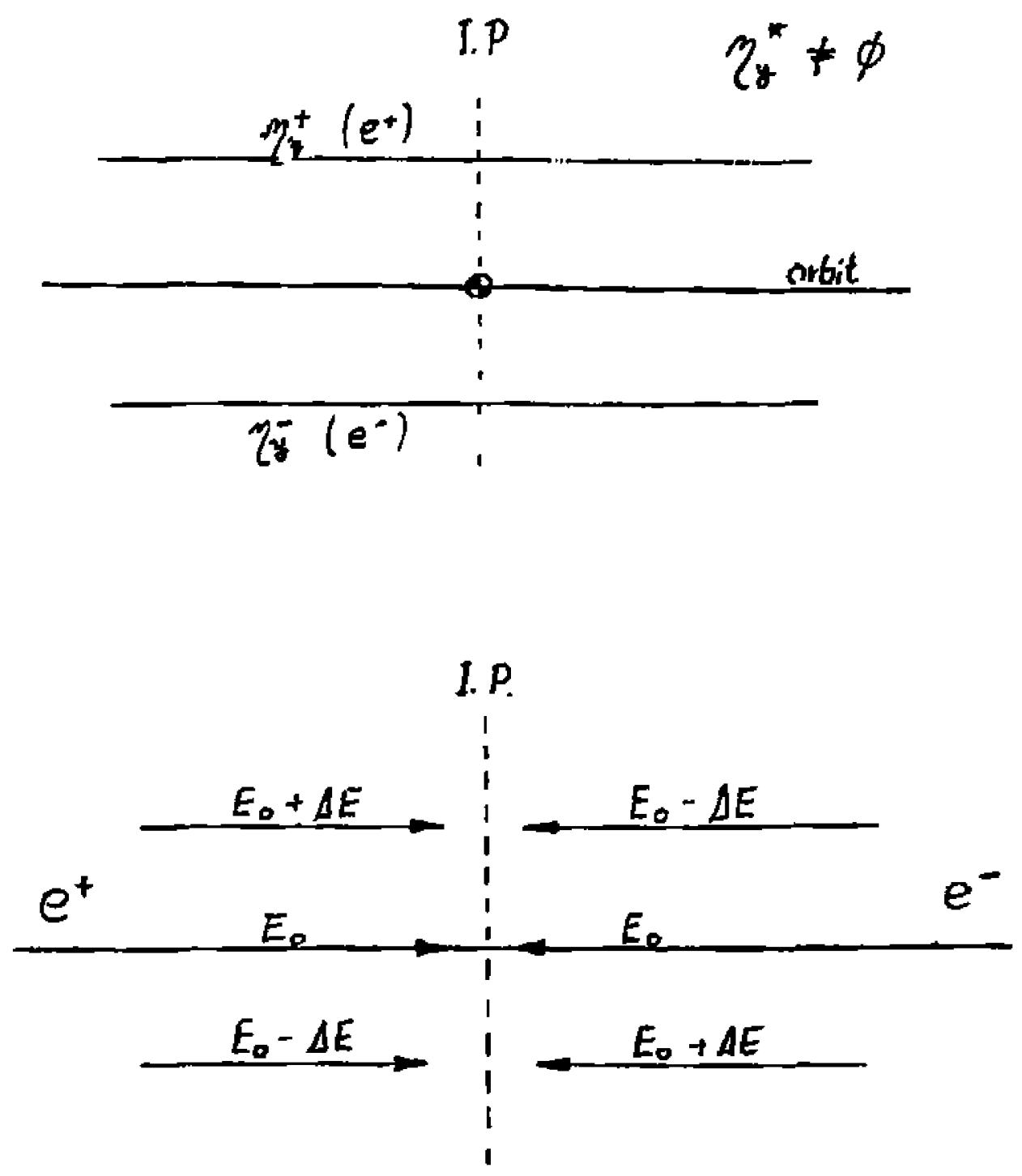

Fig. 1 


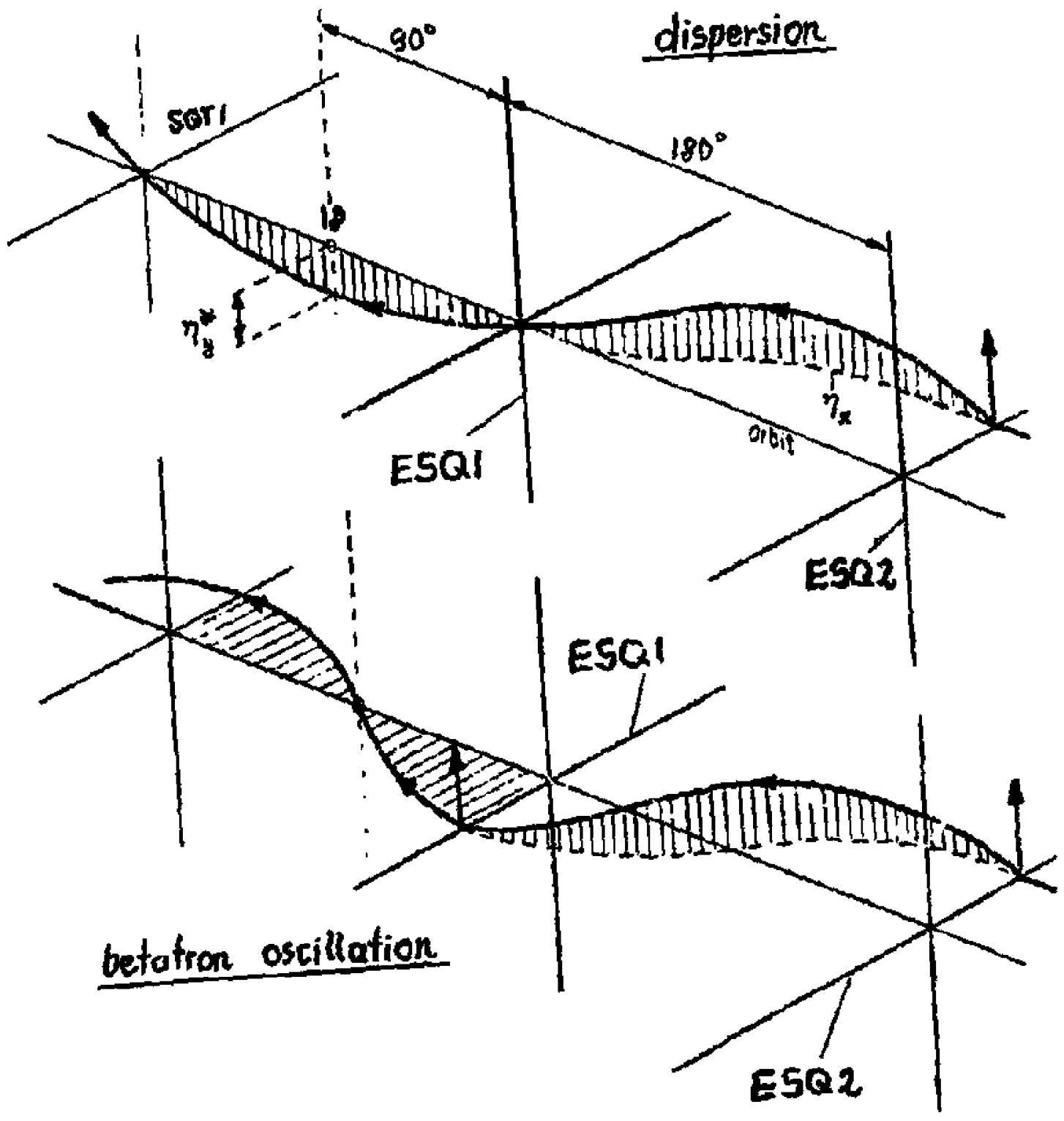

Fig. 2 


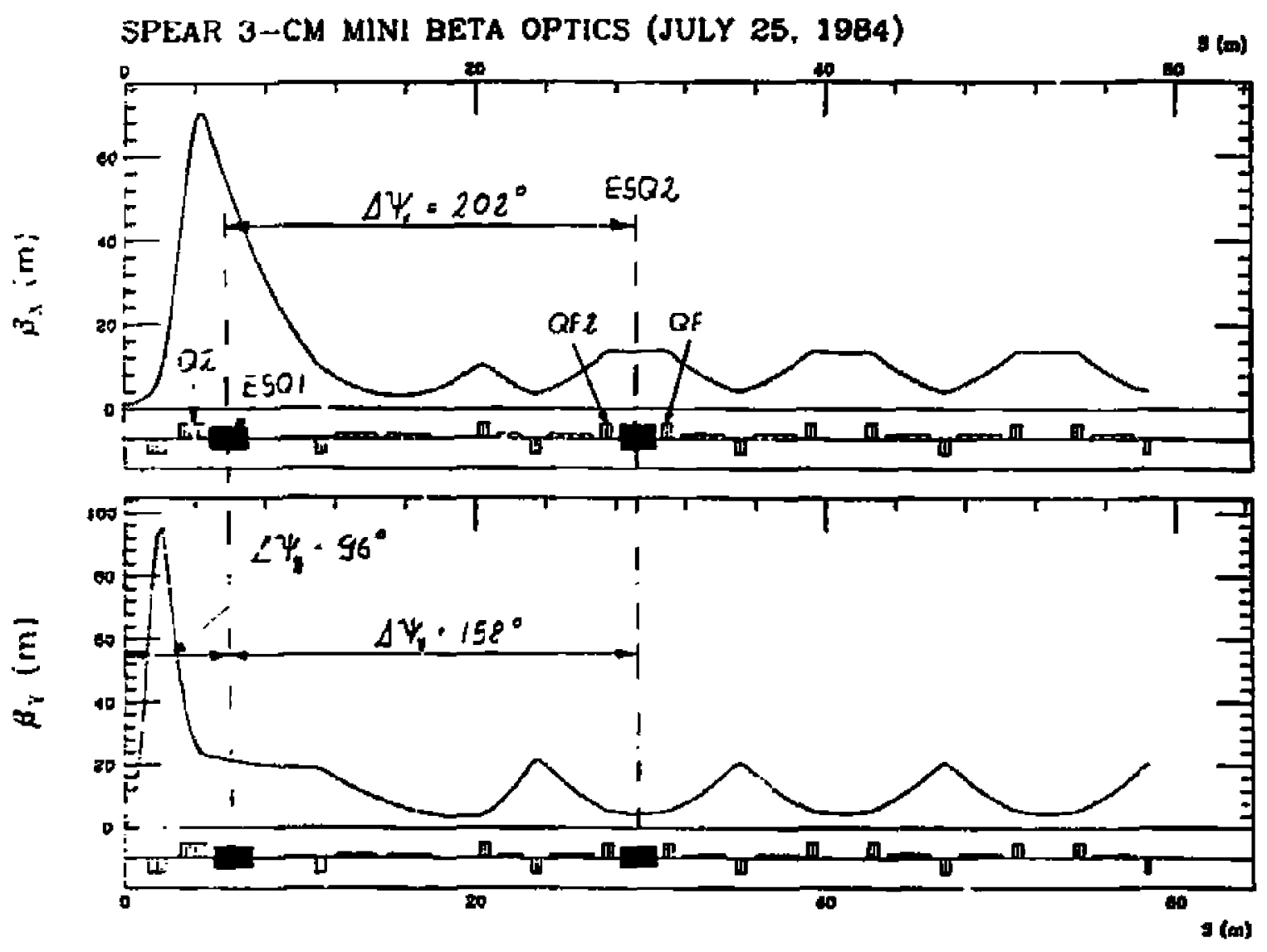

Fig. 3 

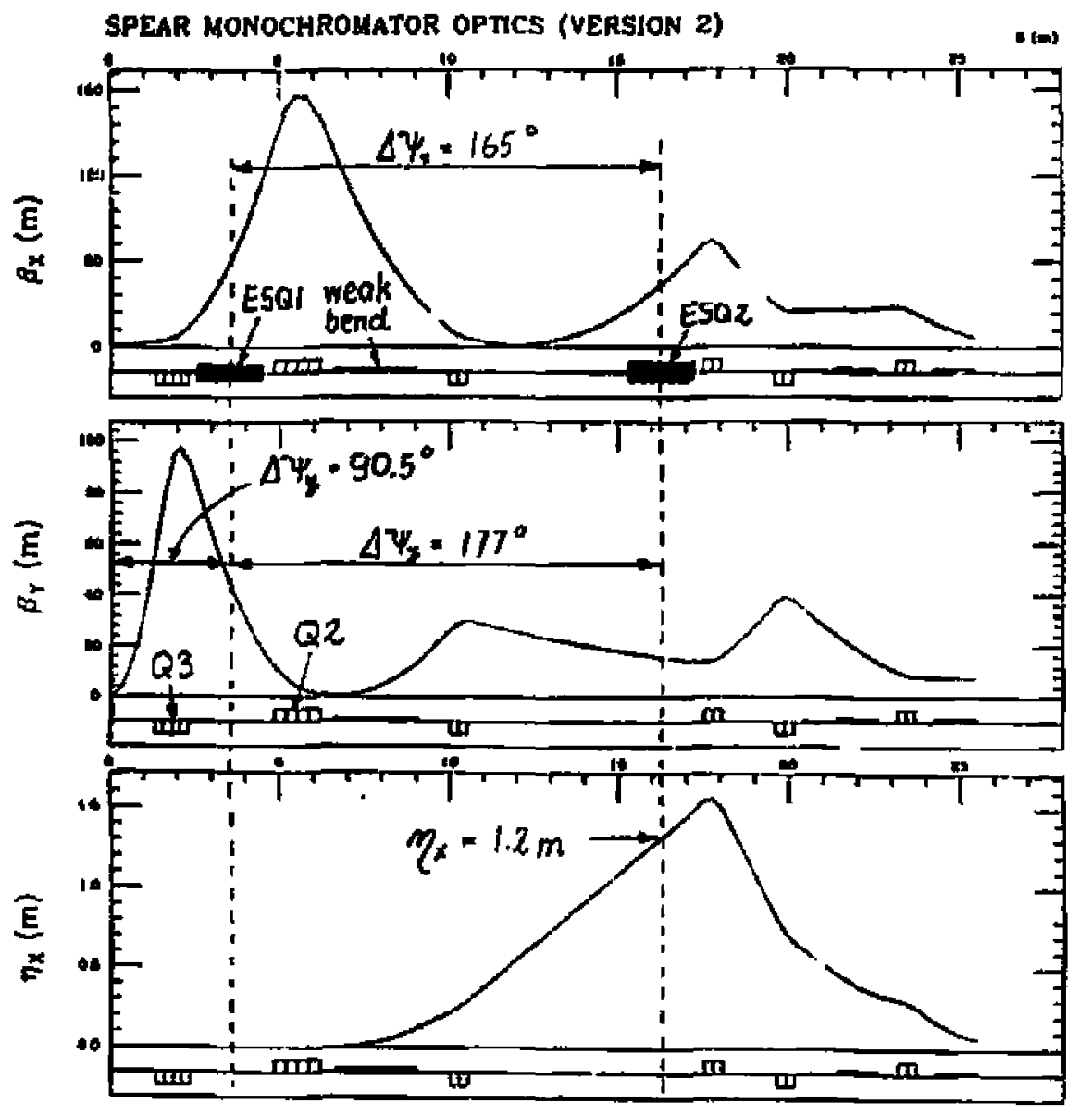

Fig. 4 


\section{DISCLAIMER}

This teport was prepared as an account of work sponsored by an agency of the United States Government. Neither the United Stutes Government nor any agency thereof. nor any of taeir employees, makes any warranty, express or implied, of assumes any legal liability of responsibility for the aceuracy. completeaess, or usefulness of any infomation, apparatus, product, $c$ is process disclosed, or represents that is use would not infringe privately owned rigbts. Reference herein to any specific commercial product, process, or service by trade name, trademark, manufacturer, ot otherwise does nol necessarily constitute or imply its endorstment, recommendation, or favoring by the United States Govemment or any agency thereor. The views and opinions of authors expressed herein do not necessarily state or ceflect those of the United States Government or any agency thereof. 